\title{
EDUCACIÓN PARA LA DEMOCRACIA EN LAS ESCUELAS: UN DESAFÍO PENDIENTE
}

\author{
Marcia Prieto \\ Instituto de Educación, Universidad Católica de Valparaíso, Chile
}

\section{LA EDUCACIÓN PARA LA DEMOCRACIA Y LA ESCUELA}

Una de las funciones de la escuela, es, entre otras, educar para la democracia, y está referida al desarrollo de las capacidades y habilidades para una convivencia social fundada en la solidaridad, la participación y el respeto, entre otros valores. Esta función es crítica para efectos del desarrollo de la autoconciencia y la responsabilidad social, dado que permitirá a los estudiantes participar como sujetos solidarios y críticos, en la escuela y en la sociedad. Al respecto el director general de la UNESCO (1993) afirmaba que la educación para la democracia implica el empoderamiento de todos los individuos para participar activa y responsablemente en todos los ámbitos de la vida política y social.

Esta función democratizadora cruza transversalmente y compromete todos y cada uno de los procesos escolares, incluyendo el sistema de relaciones que en ella se tejen, los procesos curriculares que se implementan y las estructuras de poder institucionales. Requiere de la validación de los significados de los actos y prácticas que en ella se experimentan y convoca a alumnos, profesores y administrativos a participar solidaria y colaborando en la tarea de construir un orden social democrático.

La escuela puede cumplir esta función a través de la organización e implementación de prácticas educativas que fomenten procesos deliberativos y la participación activa conducente al desarrollo integral de los estudiantes y al cultivo de los valores democráticos. En este contexto, el escuchar y fomentar la expresión de sus voces estudiantiles es importante e imprescindible dado que los son los actores y protagonistas centrales de este proceso y la calidad de sus experiencias escolares constituye un aspecto clave de su formación integral.

\section{SENTIDO DEL PROTAGONISMO DE LOS ESTUDIANTES}

Ser protagonista del proceso formativo implica ubicarse en el centro de este y desempeñar activamente las funciones centrales del mismo. Este hecho interpela a profesores y directivos a fomentar formas variadas de participación, dado que constituye uno de los ejes articuladores de las prácticas sociales en la escuela y promueve y garantiza el desarrollo de los estudiantes. Si estos operan activa, deliberada y organizadamente pueden tomar mayor conciencia del sentido y derivaciones de su participación y podrían transformarse en los verdaderos protagonistas del proceso.

Una de las formas más simples de participación de los estudiantes lo constituye la expresión de sus voces. Sin embargo, se ha desestimado sistemáticamente el derecho y necesidad que tienen de hablar por sí mismos y sus voces han permanecido separadas de los problemas que los aquejan. La voz difunde los significados y perspectivas más profundas de las personas y en el caso de los estudiantes, expresa y afirma sus propias identidades y refleja sus propias comprensiones y expectativas respecto del mundo escolar y la 
realidad que están viviendo (Fielding y Prieto, 2000). En ese sentido desconocerlas o descalificarlas impide la posibilidad de recuperar sus identidades y así comprender sus historias, lenguajes y cultura. Sin embargo, como lo afirma Juan Bautista Martínez Desconocemos lo que piensa y dice el alumnado como consecuencia de que no lo escuchamos. Los estudiantes se expresan continuamente y narran sus vivencias de manera bastante natural. Sin embargo, no escuchamos lo que dicen. No utilizamos criterios o formas estratégicas para poder atender al contenido de las expresiones del alumnado ni llegamos a considerarlo como un contenido importante para la relación pedagógica (1998:56)

Escuchar sus voces y comprender sus necesidades, expectativas y problemas no es fácil dada la gran distancia existente entre el lenguaje de los alumnos y el de los adultos, y las diversas estructuras cognitivas de cada cual para aprehender la realidad. Esta situación dificulta la interpretación de los significados asignados por los estudiantes y los adultos tienden a desconocerlos y/o ignorarlos. De hecho, aún cuando las escuelas, en ocasiones, utilizan un discurso participativo, a la hora de escuchar los planteamientos de los alumnos, surgen las más variadas djeciones para no hacerlo: se alude a una supuesta inexperiencia para representar debidamente sus perspectivas, a su incierta responsabilidad para asumir compromisos, a su ineptitud para pensar como corresponde o a su incapacidad para identificar sus problemas (Fielding y Prieto, 2000). En otras palabras, se les infantiliza y estigmatiza aplicando una supuesta ideología de la inmadurez (Grace, citada por Ruddock, et al. 1997)

El favorecer su participación es crucial, pues no sólo apoya la realización de actividades sino que también la adquisición de responsabilidades en el proyecto escolar, convirtiéndose los alumnos en agentes de su propia formación y no simples destinatarios de la acción educadora (Santos Guerra,1995). Numerosos estudios reportan que la participación de los estudiantes, cualquiera sea su edad, constituyen principios orientadores de escuelas que desarrollan en sus alumnos sus capacidades creativas, incentivan su participación en la toma de decisiones y la generación demandas, favorecen la producción de comentarios analíticos y constructivos, y valoran sus acciones cuando les asignan responsabilidades (Mena, Prieto \& Egaña, 1998; Ruddock et al., 1996; Saken,1989, Thiessen,1998). Es en este contexto que se inscribió el Proyecto Educación para la democracia en las escuelas.

\section{EL PROYECTO. EDUCACIÓN PARA LA DEMOCRACIA EN LAS ESCUELAS ${ }^{l}$}

Este proyecto se desarrolló a partir de una investigación- acción con profesores de la universidad y estudiantes para conocer y comprender, desde los estudiantes, las percepciones y compresiones de sus pares sobre la democracia en la escuela. Se desarrolló a partir de los típicos ciclos de auto reflexión que se expresan en la planificación, acción, resultados y análisis. Dado que este diseño está comprometido con un paradigma emancipatorio, en el que la responsabilidad del proceso está ubicada en el equipo mismo de investigación, y es el que toma la responsabilidad de la reflexión y la acción, el supuesto valórico que iluminó este diseño era ayudar a las personas a ayudarse a sí mismas a mejorar. Por lo tanto, esta investigación-acción, usó el análisis evaluativo, y los hallazgos para promover el mejoramiento y auto determinación de los estudiantes.

Wilfred Carr y Stephen Kemmis (1988) sostienen que la realidad social está constituida por categorías de personas con representaciones significados específicos, en consecuencia sólo es posible 
comprenderla desde los mismo actores involucradas. Dado que en este proyecto se quería conocer y comprender genuinamente las significaciones de los estudiantes respecto de la democracia era fundamental contar con la participación de estudiantes investigadores dado el papel crucial que cumplirían como representantes genuinos de sus propias voces en la decodificación del uso y comprensión del lenguaje que les es propio. De esta manera, su incorporación permitió penetrar en el mundo personal de los estudiantes, decodificar sus propias interpretaciones respecto de las situaciones y develar sus significados e intenciones

Los estudiantes investigadores participaron activamente en todas las etapas del proyecto, desarrollando y ejercitando sus capacidades creativas; promoviendo la generación de sus demandas especificas; desarrollando la habilidad para solucionar sus problemas; estimulando la producción de materiales curriculares; suscitando comentarios analíticos y constructivos y comprometiendo su participación en la toma de decisiones.

El proyecto comportó dos momentos: el primero identificó lo que los/as estudiantes percibían acerca de la democracia y el otro contempló el diseño de un programa que se implementó en sus respectivas escuelas. Para lograr lo anterior se constituyó un equipo de investigación que incluyó 8 alumnos, 4 investigadores de la universidad y un consultor internacional.. Una vez constituido el equipo se capacitó a los estudiantes investigadores en técnicas de recolección de información la que implicó la realización de 34 entrevistas individuales y 4 grupos focales. En este trabajo se da cuenta de las percepciones de los estudiantes respecto de la vivencia de la democracia en sus escuelas.

\section{RESULTADOS}

La información recogida por medio de las entrevistas individuales y grupales proporcionó evidencias de cómo los estudiantes perciben y viven la democracia en la escuela. Estas evidencias reflejan, por una parte, un desconocimiento, en un gran grupo de estudiantes del significado, implicancias y aspectos implícitos de la democracia en la escuela y por otro, una ausencia de ésta en los procesos formativos, evidenciando carencias y dificultades para el logro de una real vivencia. De los análisis realizados de esta información surgieron algunos aspectos que parecen importantes de destacar:

\section{NECESIDAD DE CONSTRUIR UN ORDEN SOCIAL DEMOCRÁTICO}

Los alumnos del estudio perciben que ingresan y viven en instituciones ordenadas sobre una lógica que no permite la construcción conjunta, la discrepancia o sus propuestas sino que, por el contrario, propicia y exige el sometimiento a lo ya establecido: el colegio es como una aristocracia, sostenía una alumna. Lo que se promueve, más bien, es la obediencia, el sometimiento y adscripción al sistema escolar en el que todo esta predeterminado, afirmaba un alumno, porque, como lo sostenía otro, todas las decisiones se toman en la oficina. Al respecto, Santos Guerra (1995:134) sostiene que si todo esta determinado, si solo importa el sometimiento a la norma, si lo único de lo que se trata es de cumplir lo prescrito y de aprender lo que oficialmente se enseña, no se arriesga ni se construye nada. Fomentar el acatamiento, el cumplimiento del deber y el temor resulta anacrónico pues representan actitudes democráticamente indeseables dado

\footnotetext{
${ }^{1}$ Proyecto Fondecyt 1990634
} 
que se establece una relación de dominación-obediencia que impide la generación de procesos de colaboración y reciprocidad.

De este modo, pareciera importante que la escuela practique la democracia dado que, por una parte, es la encargada de formar a las nuevas generaciones para participar en la sociedad y por otro, dado que los aspectos constitutivos del proceso educativos son también los propios de una democracia. Así lo plantea Josep Puig Rovira: La escuela ha de preparar a sus alumnos y alumnas para que sean capaces de asumir en el futuro las ideas y hábitos democráticos (Puig Rovira,1996:28). Pero para que ello se produzca se requiere una legitimación de los procesos que en ella se viven, se validen los significados de los actos y prácticas, convocando a alumnos, profesores y administrativos a participar solidaria y colaborativamente en esta tarea. La escuela, por lo tanto, no puede ser una organización en la que todo está preestablecido, obstruya la actividad de los alumnos e impida que ellos expresen sus voces. Si estos no tienen voz y sus inquietudes y propuestas son desestimadas, todo les será ajeno, extraño y la mayoría de las veces incomprensible. En consecuencia, para que las escuelas sean democráticas, se requiere, entre otros aspectos, fomentar la participación a partir del dialogo permanente, el debate abierto, y la crítica efectiva (Santos Guerra,1995).

\section{LA PARTICIPACIÓN: EJE ARTICULADOR DE LA VIVENCIA DEMOCRÁTICA}

La mayoría de los estudiantes entrevistados opinan que la participación se vive con actos democráticos, como la elección del centro de alumnos o cuando uno elige al presidente, a las autoridades del curso. Se podría asumir que tienen una conceptualización parcial, que la ritualizan en elecciones sin incorporar todas sus dimensiones. La participación implica, por un lado, la posibilidad de formar parte de un grupo expresando opiniones, cooperando en una actividad, situación u oportunidad. Por otro, se refiere a la posibilidad de hacerse parte de algo, la que se materializa en la capacidad de tomar decisiones acerca del propio destino y muy especialmente de las situaciones que les afectan en el contexto de su comunidad escolar. En efecto, a participación representa un proceso de comunicación, decisión y ejecución que permite el intercambio permanente de conocimientos y experiencias y clarifica el proceso de toma de decisiones y compromiso de la comunidad en la gestación, programación y desarrollo de acciones conjuntas (Murcia, 1994:15)

Ahora bien, la forma más primaria de participación la constituye la expresión de opiniones lo que, en la escuela democrática, se transforma en el medio de expresión y comprensión de los significados propios; favorece los intercambios de significados conducente al entendimiento mutuo y permite a los estudiantes comunicar, por sí mismos, lo que piensan, experimentan y desean. No obstante, a través de las respuestas de los estudiantes hemos identificado que ellos están muy conscientes que sus voces no son escuchadas y menos fomentadas. Tal como sostiene un alumno: muchas veces no somos tomados en cuenta. Entonces ¿a qué venimos? A pescar el cuaderno y escribir. Ellos perciben que los ignoran y desatienden sus dificultades para aprender: si a los alumnos les va mal, no les importa y siguen haciendo las clases igual.

La participación apunta al ejercicio permanente del diálogo, a la emisión de opiniones respecto de lo que se hace y no se hace en las escuelas, a la reflexión critica respecto de las normas y las consecuencias de su transgresión, al compromiso para la acción conjunta y la aceptación de las responsabilidades al 
respecto. Una participación activa implica operar sobre la base del pleno conocimiento y conciencia del contenido y alcance de las comprensiones y significaciones mutuas, de la misma manera que se subentiende la existencia de una noción definida de las formas, medios, alcances y oportunidades de las acciones a emprender conjuntamente. En otras palabras, debe ser una participación basada simultáneamente en el dialogo y en la realización de acuerdos y proyectos (Puig Rovira:20)

Ahora bien, las aulas escolares constituyen el lugar donde en forma intencionada se desarrollan los procesos de enseñanza y aprendizaje y en consecuencia se vive o no la democracia. Ya se mencionaba que los principios que informan la democracia son también aspectos constitutivos de la relación pedagógica En efecto, en las aulas se produce el encuentro interrelacionado de contenidos, personas, recursos, actividades y objetivos diversos que interactúan entre sí y se afectan recíprocamente; de esta manera, la naturaleza y calidad de la relaciones entre estos elementos constituiría la diferencia entre practicar o no la democracia.

Los planteamientos de los alumnos del estudio aluden a una forma de interacción en el aula notoriamente vertical como resultado de un ejercicio del poder bastante fuerte: Yo veo a los profesores arriba y a los alumnos abajo, hay como una diferencia y aquí es muy notoria, yo la siento así. Reclaman que sus voces y propuestas no son escuchadas dado que la voz del pueblo, que son los alumnos, es la menos escuchada. Los que toman las decisiones son los de arriba, sostenía un alumno. Plantean que los profesores son muy represivos y la mayoría de las veces no aceptan tu opinión. Aún cuando en ocasiones, se les invita a expresar o proponer algo, al final, lo que plantean no tiene ninguna repercusión y todo sigue igual y sus respuestas no tienen trascendencia real. Una estudiante destacaba: Me gustaría que nos preguntaran la opinión y. que lo que se habla tenga repercusión. Otra alumna sostiene: en una ocasión, hicimos una actividad donde teníamos que decir qué pensábamos. Pero, de qué sirvió eso? No sirvió de nada, si los profesores lo leyeron no lo tomaron en cuenta para nada, pues todo siguió igual. Se podría asumir que los estudiantes demandan la recuperación de sus voces y desean ser reconocidos como sujetos éticos capaces de aportar a la construcción del bien común (Alvarado y Ostina, 1998)

Los estudiantes, por lo tanto, experimentan cotidianamente la forma rígida que adquieren las relaciones al interior del aula y no pueden, en consecuencia, desarrollar su autonomía como corresponde. De hecho, perciben, mas bien, una contradicción profunda entre lo que se dice y lo que finalmente se hace. Sostienen que aún cuando parecen tener un discurso asociado al desarrollo de la autonomía, en verdad, lo que hacen la mayoría de las veces, es reprimir y coartar sus voces e iniciativas e ignorar argumentos. Deben simplemente obedecer, aceptar las decisiones de los profesores y callar sus propuestas u observaciones; deben adaptarse a lo que se les ordena, aprender a complacer a los profesores o a comportarse de una manera distinta con ellos, a fingir, como lo decía una alumna. Esta situación de fingir o sencillamente no acatar lo que solicitan los profesores es una estrategia de resistencia a la imposición que puede llevar por peligrosos camino de la descalificación y indiferencia, tal como lo afirma un alumno: yo trato de alejarme de lo que digan los profesores y vivir en un mundo separado para no tener problemas.

Es sabido que las relaciones al interior de las escuelas están mediatizadas por una estructura de poder que reprime y coarta las iniciativas de los estudiantes, dado su tradicional formato jerárquico. El estilo que predomina es autoritario, rígido, heterónomo, donde la autoridad es un valor que deben ejercer los maestros y maestras en la institución para generar y mantener el respeto de los educandos (Alvarado y Ostina, 1998:153). De este modo los alumnos no pueden sentirse parte de lo que hacen y tomar conciencia 
de las responsabilidades que sus decisiones implican. Si no lo hacen, no desarrollan su autonomía y por el contrario se forman desde una heteronimia que les empequeñece y anula como personas.

Pareciera importante, entonces, revisar como se vive cotidianamente en las escuelas, intentando disminuir el desbalance existente en las relaciones en su interior, y favorecer así la participación estudiantil en la construcción de un orden social fundado en normas de convivencia democráticas. Este orden social proporcionaría el marco de actuación para vivirlas, y comprometerse con ellas, dado que el no hacerlo conspira para la generación de la autofundación (Freire, 1994). En efecto, un orden autofundado requiere la constitución de un marco regulativo de actuación en el que todos los actores participen en la toma de decisiones, generen un sistema de representación legitimada, construyan una comunidad en la que todos participen con responsabilidad en los destinos de la misma.

Ahora bien, para que los alumnos participen y expresen de sus voces, es necesario desarrollar las habilidades analíticas y críticas para discernir y escoger. En efecto, el reporte del UNDP (1992:26) destaca que el propósito del desarrollo de la persona es ampliar el rango de sus opciones. Si no son libres para escoger, todo el proceso se vuelven una parodia. Así las personas pueden trasladarse desde una posición de considerarse objetos a quienes las cosas le pasan hacia una posición de verse como sujetos, con el derecho, la habilidad y la responsabilidad de participar en las decisiones que afectan sus vidas (Shannon,1993). El desarrollo de estas habilidades ayudará a los estudiantes a pensar y actuar autónomamente, a resolver problemas y conflictos y desarrollar la conciencia social y la autoconciencia para ejercer su libertad de actuar y pensar (Barber, 1992, Darling-Hammond, 1996; Dayton, 1995; Martínez, 1995).

Los profesores tienen todo el poder para desarrollar estas habilidades pues son quienes deciden como realizan sus clases y si demandan o no una participación activa de sus estudiantes. Por lo tanto, como sostiene Dewey, (1972) es preciso promover en la escuela la resolución de los problemas prácticos, morales y sociales a partir de la deliberación colectiva y el debate racional conducentes a decisiones y actividades conjuntas, dado que constituyen formas de trabajo que favorecen la toma de posiciones, la defensa de las ideas, el respeto y la tolerancia a las ideas de los otros

\section{LA RESOLUCIÓN DE CONFLICTOS Y LA TOLERANCIA A LA DIVERSIDAD: ELEMENTOS CRÍTICOS PARA UNA CONVIVENCIA DEMOCRÁTICA}

La paz en democracia no se construye eliminado al otro, sino que se construye resolviendo pacíficamente los conflictos. En una democracia no existen los enemigos sino que personas que piensan distintos y que tiene ese derecho. La gran mayoría de los estudiantes conceptualizó la democracia como el derecho a expresar libremente sus ideas. Esta representación parcial de democracia, es un derivado de su necesidad de expresar sus opiniones e iniciativas sin temor a ser descalificados o reprimidos: muchas veces tenemos que bajar la cabeza y uno se retrae de decir cosas que le gustaría decir por el temor a las represalias, destacaba un alumno y otro sostenía que la democracia está referida a que todos puedan opinar sin necesidad de tener represiones, ni nada de eso. Tampoco discriminados sobre la base del cargo, edad, pensamiento político o religiosos. Ellos notan que sólo se considera la opinión de los que tienen más importancia en el curso, el presidente, el tesorero; del mismo modo advierte que cuando nuestro 
pensamiento político o religioso se encuentra en minoría, no encontraremos espacios en nuestra comunidad para poder expresar nuestra opinión y poder formar parte de la sociedad en igualdad de condiciones.

Se podría deducir que la ética propia de los procesos formativos, que establece que la edad no puede constituirse en un elemento de discriminación y descalificación, está aparentemente ausente, según sus percepciones. Ellos insisten que no quieren ser considerados enemigos a los que hay que destruir psicológicamente, a través de la descalificación, la indiferencia o el etiquetamiento y tienen razón pues la vida democrática se desploma cuando predomina la falta de respeto y la desconfianza, cuando no se escuchan los puntos de vista de los demás o cuando no se admite los errores (Meir, 1995)

Los conflictos surgidos en la vida cotidiana de una escuela se pueden resolver por medio de la deliberación conjunta, la expresión de ideas y la sustentación y defensa de posiciones. En esta perceptiva, los alumnos son actores que tiene derecho a expresar y defender sus ideas en un contexto de respeto y tolerancia De esta manera, no es extraño que los alumnos hayan nombrado la tolerancia como uno de los aspectos básicos para la existencia y desarrollo de la democracia y reclaman que es necesario Aceptar a la persona, tal como es, tolerar sus cosas malas y agradecerle las positivas. Sin embargo, en la escuela ellos experimentan que a menudo la opinión de otra persona ofusca, enoja y hasta ahí nomás llega la tolerancia. Yo por lo menos no la vivo así y trato de alejarme de lo que digan los profesores. Estas observaciones llevan a pensar en la necesidad de estimular y desarrollar la tolerancia y el respeto a la diversidad, como una manera de resolver muchos conflictos y erradicar muchas violencias. Es un hecho que la escuela forma desde y en la diversidad y esta situación apoya el desarrollo de la tolerancia pues exige el respeto a la diferencia del mismo modo como la vivencia de la justicia requiere la superación de la discriminación (Santos Guerra, 1995)

En este punto se hace necesario establecer algunas precisiones. Por una parte, es necesario reconocer existe una diversidad legítima y otra ilegítima. La tolerancia respeta aquella diversidad legítima y condena la ilegitima. Sin duda que estos conceptos están marcados por un relativismo que a veces lleva a confusiones y surge la duda respecto a ¿Cómo identificar lo que es legítimo de lo que no lo es sin caer en totalitarismos intolerantes?. ¿Cómo resolver las diferentes interpretaciones que surgen en la vida cotidiana de la escuela sin caer en autoritarismo o neutralismos donde todo vale? Los estudiantes no quieren eso pues están conscientes que los límites forman parte de la convivencia. Un alumno sostenía que al expresar lo que quiero no puedo pasar por encima de todas las personas. Otro decía que todos pueden opinar, dar a conocer sus ideas, pero sin herir a las demás personas. Es decir, reconocen y aceptan que los límites son importantes para que todos puedan convivir y crecer.

\section{CONCLUSIONES}

Las conclusiones de este trabajo conjunto con estudiantes están relacionadas con varios aspectos. Tienen que ver con la necesidad, por una parte, de promover el protagonismo de los estudiantes y por otro, de aprender a trabajar con ellos. 


\section{PROMOVIENDO EL PROTAGONISMO DE LOS ESTUDIANTES}

Promover el protagonismo de los estudiantes implica ubicarlos en el epicentro de sus procesos formativos y reivindicar su calidad de sujetos. En este contexto, la escuela no puede ser una organización en la que todo esta preestablecido, ni menos significar a los estudiantes como simples objetos de acciones técnicas planificadas para el logro de fines institucionales.

Uno de los aprendizajes surgidos del desarrollo del proyecto, apunta a reconocer que para lograr este protagonismo es preciso fomentar tanto su participación activa y constante en los procesos relacionados con su desarrollo, como su involucramiento en los asuntos escolares de interés común. Los estudiantes necesitan darse cuenta que son reconocidos como personas competentes, con ideas y convicciones, capaces de tomar decisiones y de apoyar las acciones que ellos consideran importantes en el contexto de una comunidad de apoyo mutuo. Si no tienen voz y sus inquietudes, proyecto y propuestas para la construcción de una mejor comunidad son desestimadas sentirán que todo les es ajeno, extraño y la mayoría de las veces incomprensible.

Lograr el protagonismo implica, por lo tanto, fomentar y tomar en cuenta sus iniciativas, diseñar e implementar formas de trabajo cooperada y próximas a sus vidas cotidianas, respetar sus diversidades y preferencias e incorporarlos en la construcción de su comunidad escolar. Sin embargo, la participación no es fácil ni se produce espontáneamente; es preciso habilitar a los estudiantes para ello a partir del desarrollo de la autonomía o capacidad de autorregulación que permite a la persona construir sus propias visiones, defender sus posiciones y hacer coherente lo que piensa con lo que hace.

También es necesario desarrollar la habilidad para razonar y comprender críticamente a partir de la práctica sostenida del diálogo.. Este permite a la persona intercambiar opiniones, razonar sobre distintos puntos de vista, intentar llegar a un entendimiento entre todos y resolver los conflictos o disonancias de manera pacífica. En efecto, los diferentes conflictos que surgen en la vida cotidiana de la escuela se pueden resolver por medio de la expresión de las ideas, la deliberación colectiva, el entendimiento racional y la sustentación y defensa de las posiciones. Estas prácticas permitirían manejar el conflicto y la diversidad, prefigurando lo que pueden ser nuevas formas de comportarse y vivir en sociedad y descartando, así, el uso de la violencia, la descalificación e indiferencia, el etiquetamiento y la confrontación destructiva material y psicológica del otro diferente.

\section{APRENDIENDO A TRABAJAR CON LOS ESTUDIANTES}

Otra conclusión importante está relacionada con tomar conciencia que es necesario aprender a trabajar con los estudiantes como iguales en derecho y dignidad y por lo tanto, con capacidad para proponer y decidir respecto de los asuntos que ellos consideran importantes y que les afecta. Esta situación no se da per se, requiere escuchar y fomentar la expresión de las voces de los estudiantes e investigan con ellos. Al respecto una alumna expresaba, refiriéndose a la forma como se había trabajado en el proyecto: apreciamos nuevas formas de trabajo y personas que nos ayudan a opinar y a aprender a comunicarnos entres jóvenes y adultos. 


\section{PROMOVIENDO Y ESCUCHANDO SUS VOCES}

Se piensa que las interacciones sociales son procesos lineales de decodificación mecánica. Sin embargo, la realidad indica que existen innumerables problemas de comunicación y es difícil entender lo que los jóvenes dicen. Una de las causas de esta incomunicación se debe al desconocimiento de la vida que ellos viven en sus espacios socio-culturales y desde donde surgen sus propias construcciones. Este desconocimiento lleva a los adultos dominar e imponer una forma de ver el mundo, una forma de descubrirlo, significarlo y denotarlo. Por lo tanto, al aprender a escuchar a los estudiantes, se advierten sus diferentes situaciones biográficas, sus comprensiones y significaciones acerca de la realidad, sus distintos niveles de conocimiento para aprehenderla y interpretarla y se hace posible una verdadera relación formativa.

Dado que el proceso formativo en la escuela implica profesores y estudiantes en relación y que las significaciones deben ser negociadas y discutidas para acceder a una comprensión común, no es posible desentenderse de lo que piensan y dicen los estudiantes. De ahí la necesidad de entrar en su mundo, comprender y discutir las significaciones que regulan su vida cotidiana, y así establecer las bases comunes para una construcción conjunta.

Aprender a escucharlos es solo el primer paso que permitirá realizar una tarea formativa efectiva. En efecto, es preciso, además, ayudarles a transformase en agentes de sus propios aprendizajes y así poder utilizar lo que dicen de manera productiva (Nieto, 1993). Ello implica recuperar y valorar lo que estudiantes traen a las escuelas como contribuciones criticas para el proceso y manifestar una genuina sensibilidad para identificar as creencias que están subyaciendo tras sus reflexiones, recuperando la validez de sus contribuciones como una forma de entender el mundo desde distintas significaciones y alcanzar comprensiones comunes. Para lograr lo anterior es indispensable que los actores se valoren y respeten entre sí, y construyan una comunidad que favorezca el compromiso, aprendizaje y desarrollo mutuo (Darling -Hammond, 1996; Ranson et al., 1996). Si se trabaja promoviendo la expresión de sus voces y comprendiendo e incorporando sus construcciones de mundo y respectivas significaciones, se logrará visualizar sus posibilidades y habilidades para colaborar en la construcción de su comunidad escolar.

\section{APRENDIENDO A TRABAJAR COLABORATIVAMENTE}

Una forma de trabajar con los alumnos es incorporarlos en trabajos colaborativos dado que les permite confrontar sus propios modos de operación con el de otros, aprender a discutir argumentos, respetar las ideas de otros y escuchar sin descalificar o sentirse personalmente atacado cuando otro tiene una opinión distinta. También les permite comprobar, a partir de las discusiones, que existen formas de trabajo que van más allá del simple proceso de sumar los aportes individuales pues advierten que es posible generan un cuerpo de conocimiento distinto, con los aportes de cada uno integrados en un todo más amplio. Del mismo modo, los aprendizajes compartidos apuntan tanto a la participación del alumno en la construcción del conocimiento a partir de la actividad que desarrolla, como al aprendizaje a partir de cooperar con otros. Lo anterior tiene como resultado un potenciamiento de las capacidades individuales para responder a sus propias expectativas y tomar decisiones colectivas para la acción y el cambio. 


\section{INVESTIGANDO CON ELLOS}

Otra forma específica de convertir a los estudiantes en protagonistas de sus procesos formativos es investigando con ellos. Al producirse un consorcio genuino entre el investigador y los estudiantes se les habilita para que se transformen en agentes tanto de las historias que producen como de sus propios proceso de cambio. Se abre un espacio para que se planteen respecto de qué se podría cambiar, cómo podrían contribuir al cambio, qué no esta resultando y porqué. Les permite lograr una mayor percepción respecto de sus propias habilidades y adquieren conciencia de que pueden contribuir a mejorar la escuela no sólo para ellos sino también para otros.

Asimismo, los profesores, al compartir con estudiantes logran conocerlos en un plano distinto: el de actores involucrados en la misma tarea, que tratan en su conjunto mejorar la escuela, y pueden descubrir sus capacidades propositivas y creativas. Las tareas de enseñanza se tornan menos amenazantes o tensas para los profesores pues las responsabilidades son compartidas. Las relaciones también mejoran substantivamente entre ellos pues los profesores toman en cuenta y valoran sus contribuciones, sus preferencias, ponderan sus interpretaciones, y analizan sus críticas. En definitiva, los estudiantes no sólo logran ser escuchados sino que también se les ayuda a transformarse en agentes de sus propios procesos de cambio.

Dado lo anteriormente dicho se puede concluir que es necesario resignificar el sentido de la escuela y asumirla como una agencia en la que todos se potencian y desarrollan entre todos en un ambiente participativo y comunitario. Demanda establecer relaciones entre los actores que trasciendan el aspecto técnico de simple comunicación de contenidos y órdenes y se encamine hacia la comprensión mutua de valores y significados y la interpretación recíproca de los actos propios en el contexto de la cotidianeidad.

En este tipo de escuela tanto profesores como directivos no sólo deberían escuchar a los estudiantes sino también motivarlos a participar con sus ideas, argumentos y propuestas en la construcción conjunta de la comunidad escolar. Ello interpela substantivamente a la forma como se viven los principios centrales que informan la democracia, a la articulación de las prácticas sociales que se desarrollan y a las oportunidades que se ofrecen para que los alumnos puedan expresar sus voces y tomar decisiones respecto de los asuntos que les afectan. Requiere de profesores y directivos con la disposición para compartir el poder con quienes tienen el derecho y la obligación de participar con sus voces y propuestas en los procesos escolares y no los marginen desconociendo o ignorando sus preferencias, propuestas y necesidades específicas.

Finalmente, es preciso construir un nuevo orden escolar, que tome en cuenta la realidad idiosincrásica de cada escuela, que respete las singularidades tanto personales como culturales de los actores que en ella conviven y se establezcan límites conocidos y discutidos por todos. La configuración de un orden así descrito podría llevar a la escuela a resignificar una de sus funciones más críticas: la formación de personas autónomas, respetuosas y tolerantes de la diversidad; solidarias con los destinos de su comunidad; atentas y críticas frente a lo hechos y situaciones de la sociedad en la que viven y creativas para transformar y construir un nuevo orden, plasmado en torno a los principios de igualdad, libertad, justicia y participación. 


\section{REFERENCIAS}

Alvarado S y Ospina H (1998) La escuela como escenario potencial para la construcción de la paz. Los niños y niñas como sujetos cono sujetos éticos. Etica Ciudadana y Derechos Humanos. Una Contribución a la Paz .Santa Fe de Bogotá: Magistero/ CINDE

Darling -Hammnond, L (1996) The right to learn and the advancemente of teaching: Research, policy and practice for democratic education. Educational Researcher,25, 6,pp5-17

Dewey,J(1972). Democracia y Escuela. Buenos Aires: PAIDOS

Freire, P(1994). Educación y Participación Comunitaria. Nuevas Perspectivas Críticas en Educación, Castells, M et al. Buenos Aires: Paidos Educador

Fielding, M y Prieto, M (2000) Investigando con alumnos: una experiencia práctica democrática. PAIDEAI, N²8,pp 105-126

Giroux, H.(1988) Schooling and the Politics of Ethics: Beyond Liberal and Conservative Discourses. Journal of Education, 169, 2, pp 9-33

Martinez, J (1998) Tiene el alumnado posibilidad o derecho a realizar innovaciones?. Volver a pensar la educación. Vol II. Madrid Morata

Mena,I,Prieto,M y Egañan, P (1999) Aventuras Pedagógicas de Comunidades que Aprenden. Santiago de Chile: UNICEF y Misterio de Educación Pública.

Meir, D. (1995) How our schools could be? Phi Delta Kappan,76, 5,pp369-373

Murcia, (1994) Investigar para Cambiar. Bogotá: Magisterio

Lincoln, I (1995) In Search of Students'Voices. Theory into Practice, v 34, pp88-93

Prieto, M. (2001) Mejorando la Calidad de la Educación: Hacia una Resignificación de la Escuela. Valparaíso: Ediciones Universitarias de Valparaíso

Puig, J et al. (2000)Como fomentar la participación en la escuela. Serie Pedagogía: Teoría y Práctica. Barcelona: GRAO

Reyzabal, M.V.y Sanz,A.I (1999) Los Ejes transversales. Aprendizajes para la Vida. Madrid: Escuela Española

Ruddock, J. et al. (1996 ) "Student Voices: what can they tell us as partners in change? en K. Scott y V.N. Trafford (eds.) Partners in Change: Shaping the Future, Middlesex: University Press

Sandoval, P.(1999) Descentralización en la Construcción Curricular: Reforma Educacional Chilena. Boletín de Investigación Educacional. PUC, vol.14,pp370-386

Santos Guerra, M. (1995)Democracia escolar o el problemas de la nieve frita. Volver pensar la educación. Vol I. Madrid: Morata

Shannon,P. (1993): Developing democratic Voices. The Reading Teacher,47,2,pp86-94

SooHoo, S. (1993): Students as Partners in Research and Reestructuring Schools. Educational Forum, 57,pp386-393

Sotelo, I. (1995). Educación y Democracia. Volver a pensar la educación(vol. I)Madrid: Morata

UNESCO (1993). Discurso Director General de la UNESCO. Congreso Internacional sobre Educación, Derechos Humanos y Democracia. Montreal. Human Rights Teaching ,8,pp26-33

UNDP (1993) .Human Development Report. New York:UNDP 


\title{
Contactar
}

Revista lberoamericana de Educación

\author{
Principal OEI
}

